\title{
Estudo fitoquímico e avaliação da toxicidade frente a Artemia salina e da atividade antimicrobiana de Calycorectes psidiiflorus (O. Berg) Sobral, Myrtaceae
}

\author{
Elaine A. Domingues, ${ }^{* 1}$ Celso V. Nakamura, ${ }^{2}$ Maria C. de Souza, ${ }^{3}$ Tatiane S. Teixeira, ${ }^{1}$ \\ Juliana L. B. Peixoto, ${ }^{1}$ Maria H. Sarragiotto, ${ }^{1}$ Gentil J. Vidotti ${ }^{\dagger, 1}$
}

\begin{abstract}
${ }^{1}$ Departamento de Química, Universidade Estadual de Maringá, Av. Colombo 5790, 87020-900 Maringá-PR, Brasil ${ }^{2}$ Departamento de Ciências Básicas da Saúde, Universidade Estadual de Maringá, Av. Colombo 5790, 87020-900 Maringá-PR, Brasil

${ }^{3}$ Departamento de Biologia, Universidade Estadual de Maringá, Av. Colombo 5790, 87020-900 Maringá-PR, Brasil.
\end{abstract}

\begin{abstract}
RESUMO: O estudo fitoquímico das folhas de Calycorectes psidiiflorus (O. Berg) Sobral, Myrtaceae, resultou no isolamento e identificação de: sesquiterpeno [8-hidroxicalameneno (1)], triterpenos [ $\alpha$-amirina (2a) e $\beta$-amirina (2b)], flavonóide [3- $O$ - $\alpha$-ramnopiranosil-7- $O$ - $\beta$ glucopiranosil canferol (3)], e alcalóide [1,2,3,4-tetraidro-1-metil- $\beta$-carbolina (4)]. As estruturas das substâncias isoladas foram elucidadas com base nos seus dados de RMN em comparação com os da literatura. A substância 8-hydroxicalameneno apresentou atividade antibacteriana (MIC = 7,8 $\mu \mathrm{g} / \mathrm{mL}$ ) e antifúngica ( $\mathrm{MIC}=15,6 \mu \mathrm{g} / \mathrm{mL})$.
\end{abstract}

Unitermos: Calycorectes psidiiflorus, Myrtaceae, constituintes químicos, ensaios biológicos.

\begin{abstract}
Phytochemical study and evaluation of toxicity against Artemia salina and antimicrobial activity of Calycorectes psidiiflorus (O. Berg) Sobral, Myrtaceae". The phytochemical study of Calycorectes psidiiflorus (O. Berg) Sobral leaves resulted in isolation and identification of the sesquiterpene 8-hydroxycalamenene (1), triterpenes $\alpha$-amyrin (2a) and $\beta$-amyrin (2b), flavonoid 3-O- $\alpha$-rhamnopyranosyl-7- $O$ - $\beta$-glucopyranosyl kaempferol (3), and of the alkaloid 1,2,3,4-tetrahydro-1-methyl- $\beta$-carboline (4). The structures of the isolated compounds were elucidated based on their spectroscopic NMR data and comparison with those reported in literature. Substance 1 presented antibacterial ( $\mathrm{MIC}=7.8 \mu \mathrm{g} / \mathrm{mL}$ ) and antifungal (MIC $=15.6 \mu \mathrm{g} /$ $\mathrm{mL})$ activities.
\end{abstract}

Keywords: Calycorectes psidiiflorus, Myrtaceae, chemical constituents; biological assays.

\section{INTRODUÇÃO}

Calycorectes psidiiflorus (O. Berg) Sobral pertence à família Myrtaceae, que abrange cerca de cem gêneros e três mil espécies (Trease, 1983). Compreende duas subfamílias, a Leptospermoideae que reúne as espécies de frutos secos com maior concentração na Austrália, e a Myrtoideae que apresenta espécies de frutos carnosos e se concentra principalmente nas Américas do Sul e Central (Romagnolo, 2003). A subfamília Myrtoideae possui espécies com características apícolas e produzem frutos comestíveis muito apreciados pela fauna silvestre e também pelo homem, tais como: pitanga, goiaba, araçá, jabuticaba, guabiroba, uvaia, jambolão, jambo (Romagnolo, 2003).

Calycorectes psidiiflorus é conhecida popularmente como guamirim-de-riedel ou cambuí (Romagnolo, 2003) e apresenta-se normalmente como uma arvoreta, com cerca de três metros de altura, mas pode alcançar até dez metros; possui flores com pétalas brancas e frutos globosos (10-20 mm de diâmetro) de coloração vermelha quando maduros. Sua distribuição geográfica ocorre no Brasil, Argentina, Paraguai e Uruguai. As folhas de Calycorectes psidiiflorus, conhecidas popularmente no Paraguai como Ñangapiry (Schmeda, 1995), em infusão ou decocção, são utilizadas como digestivo, antihipertensivo.

O gênero Calycorectes apresenta cerca de dezoito espécies distribuídas do México até a América do Sul e que são utilizadas na medicina popular, porém poucos estudos são apresentados na literatura.

O presente trabalho tem como objetivo a investigação fitoquímica de Calycorectes psidiiflorus (folhas e galhos) visando o isolamento e identificação dos principais compostos presentes na planta, de forma a 
ampliar os estudos químicos da espécie e contribuir para os estudos quimiotaxonômicos do gênero, bem como avaliar as atividades antibacteriana e antifúngica dos extratos e substâncias isoladas.

\section{MATERIAL E MÉTODOS}

\section{Instrumentação e material cromatográfico}

Nos procedimentos de separação por coluna cromatográfica $(\mathrm{CC})$ foram utilizadas sílica gel 60 $(0,063-0,200 \mathrm{~mm})$ e sílica flash $(0,035-0,070 \mathrm{~mm})$ como fases estacionárias. Como fases móveis foram utilizados solventes puros e ou combinados, em ordem crescente de polaridade. Na cromatografia de camada delgada (CCD) e cromatografia de camada delgada preparativa (CCDP) foi utilizado sílica gel 60 GF (Merck), nas espessuras 0,3 e 0,8 $\mathrm{mm}$, respectivamente.

Os espectros de $\mathrm{RMN}$ de ${ }^{1} \mathrm{H}$ e de ${ }^{13} \mathrm{C}$ foram registrados em espectrômetro VARIAN, modelo Mercury Plus BB, $300 \mathrm{MHz},\left(300,06 \mathrm{MHz}\right.$ para ${ }^{1} \mathrm{H}$ e $75,45 \mathrm{MHz}$ para ${ }^{13} \mathrm{C}$ ).

\section{Material vegetal}

A espécie vegetal Calycorectes psidiiflorus (O. Berg) Sobral, Myrtaceae (folhas e galhos), foi coletada em novembro de 2001, às margens da planície alagável do alto rio Paraná, na região de Porto Rico-PR. Sua identificação foi feita pela $\operatorname{Prof}^{a}$. Dr ${ }^{\mathrm{a}}$. Maria Conceição de Souza, do Departamento de Biologia da Universidade Estadual de Maringá. Uma exsicata de Calycorectes psidiiflorus foi depositada no acervo do Herbário da Universidade Estadual de Maringá (HUM) sob no 9602, coletor: M. B. Romagnolo 316.

\section{Extração e isolamento das substâncias}

As folhas e os galhos secos e moídos de $C$. psidiiflorus foram, separadamente, submetidos à extração com etanol 95\%, utilizando técnicas de maceração e percolação combinadas. Após liofilização obteve-se extrato bruto das folhas e extrato bruto dos galhos de $C$. psidiiflorus, os quais foram fracionados por partição em solventes originando as frações hexânica, clorofórmica e acetato de etila. A fração hexânica foi submetida a sucessivas colunas cromatográficas $(\mathrm{CC})$ de sílica gel, empregando os eluentes hexano, clorofórmio e metanol (na forma binária e em ordem crescente de polaridade), o que resultou em sete sub-frações. A purificação da sub-fração 7.1 por cromatografia de camada delgada preparativa (CCDP, sílica gel, $\mathrm{CH}_{2} \mathrm{Cl}_{2}$, vapor de amônia) forneceu a substância 1 na forma de um líquido de coloração amarela.

A sub-fração 7.2 foi submetida a CC de sílica gel 60 com os eluentes hexano:éter etílico em ordem crescente de polaridade, e uma das frações forneceu a mistura das substâncias $\mathbf{2 a}$ e $\mathbf{2 b}$, na forma de cristais brancos solúveis em diclorometano. A quantidade obtida de $\mathbf{2 a}$ e $\mathbf{2 b}$ correspondeu a $29,51 \%$ da subfração, ou seja, $9,74 \%$ da fração hexânica e $2,38 \%$ do extrato bruto das folhas de Calycorectes psidiiflorus.

A fração acetato de etila foi submetida à $\mathrm{CC}$ de sílica gel com o eluente $\mathrm{CH}_{2} \mathrm{Cl}_{2}: \mathrm{MeOH} 2 \%$, em ordem crescente de polaridade, sendo coletadas seis frações. A subfração 5 foi submetida à CCDP, sendo utilizado como fase móvel $\mathrm{CH}_{2} \mathrm{Cl}_{2}$ :metanol $20 \%$, o que forneceu a substância 3 como um sólido amarelo.

Do extrato bruto dos galhos de Calycorectes psidiiflorus foi realizada uma extração ácido-base. A solução aquosa ácida (com solução de ácido sulfúrico pH 2) foi extraída com $\mathrm{CH}_{2} \mathrm{Cl}_{2}(3 \times 100 \mathrm{~mL})$ e então alcalinizada com hidróxido de amônio $30 \%$ até pH 10 . A solução básica foi extraída com $\mathrm{CH}_{2} \mathrm{Cl}_{2}(3 \times 100 \mathrm{~mL})$ fornecendo a fração $\mathrm{CH}_{2} \mathrm{Cl}_{2}$ básica. Esta fração foi submetida à $\mathrm{CCDP}$, usando como fase móvel acetato de etila:metanol $70 \%$ (com vapor de amônia), o que levou ao isolamento da substância 4 que apresentou fluorescência a UV $254 \mathrm{~nm}$ e teste positivo para alcaloides com revelador de Dragendorff.

\section{Ensaios biológicos}

A avaliação da toxicidade frente à Artemia salina foi realizada com o extrato bruto das folhas e dos galhos de C. psidiiflorus e com as frações resultantes de seu fracionamento. O bioensaio de toxicidade foi realizado conforme descrito na literatura (Dey \& Harbone, 1993). Os testes foram realizados em triplicata. A concentração de amostra necessária para promover a mortalidade de 50\% e $90 \%$ dos microcrustáceos $\left(\mathrm{DL}_{50}\right.$ e $\left.\mathrm{DL}_{90}\right)$ foi calculada através do logarítimo da concentração versus a média da porcentagem de mortalidade.

No estudo da atividade antibacteriana e antifúngica foi utilizado o método de microdiluição (Mclaughlin et al., 1991) para determinação da concentração mínima inibitória. Foi utilizado o extrato bruto das folhas e dos galhos de C. psidiiflorus, as frações resultantes do fracionamento do extrato bruto e as substâncias isoladas 8-hidróxicalameneno (1), $\alpha$-amirina (2a), $\beta$-amirina (2b),3-O- $\alpha$-raminopiranosil-7- $O-\beta$-glicopiranosil canferol (3) e 1,2,3,4-tetraidro-1-metil- $\beta$-carbolina (4).

\section{RESULTADOS E DISCUSSÃO}

O estudo fitoquímico das folhas e galhos de Calycorectes psidiiflorus (O. Berg) Sobral, Myrtaceae, resultou no isolamento de três substâncias puras, identificadas como 8-hidróxicalameneno (1), 3-O- $\alpha$ raminopiranosil-7-O- $\beta$-glicopiranosil canferol (3) e 1,2,3,4-tetraidro-1-metil- $\beta$-carbolina (4) e uma mistura de duas substâncias identificadas como $\alpha$-amirina (2a) e $\beta$-amirina (2b).

A substância 1 foi isolada da fração hexânica 
das folhas de $C$. psidiiflorus. No espectro de $\mathrm{RMN}$ de ${ }^{1} \mathrm{H}$ observaram-se dois singletos na região de hidrogênios aromáticos em $\delta_{\mathrm{H}} 6,40$ e 6,57 , cada um apresentando integração para um hidrogênio. Foram verificados três dubletos em $\delta_{\mathrm{H}} 0,82(J=6,9 \mathrm{~Hz}), \delta_{\mathrm{H}} 0,97(J=6,9 \mathrm{~Hz}) \mathrm{e}$ $\delta_{\mathrm{H}} 1,19(J=6,9 \mathrm{~Hz})$, cada um com integração para três hidrogênios, característicos de metilas. Foi observado também um singleto em $\delta_{H} 2,22$ com integração para três hidrogênios, sinal típico de metila ligada a anel aromático, e um singleto em $\delta_{H} 4,89$, com integral para um hidrogênio, característico de hidroxila.

No espectro de $\mathrm{RMN}$ de ${ }^{13} \mathrm{C}$, com auxílio de experimentos de DEPT, foi possível determinar a presença de quatro carbonos metílicos $\left(\delta_{\mathrm{C}} 19,8 ; 21,3 ; 21,4\right.$ e 22,3$)$, dois carbonos metilênicos $\left(\delta_{\mathrm{C}} 19,3\right.$ e 27,3$)$, cinco carbonos metínicos $\left(\delta_{\mathrm{C}} 26,8 ; 33,4 ; 43,3 ; 113,6\right.$ e 123,1$)$ e de quatro carbonos não ligados a hidrogênio $\left(\delta_{C} 126,3 ; 135,2 ; 141,4\right.$ e 153,2). Através do espectro de HMQC foi possível verificar que a substância apresenta apenas um metileno $\left(\delta_{C}\right.$ 27,3) com hidrogênios heterotópicos, correspondente aos hidrogênios $\delta_{\mathrm{H}} 1,50$ e $\delta_{\mathrm{H}} 1,96$ e ainda que as quatro metilas $\left(\delta_{\mathrm{C}} 19,8, \delta_{\mathrm{C}} 21,3, \delta_{\mathrm{C}} 21,4\right.$ e $\left.\delta_{\mathrm{C}} 22,3\right)$ têm respectivamente, $\delta_{\mathrm{H}} 0,82(J=6,9 \mathrm{~Hz}), \delta_{\mathrm{H}} 2,22$ singleto, $\delta_{\mathrm{H}} 1,19(J=6,9 \mathrm{~Hz})$ e $\delta_{\mathrm{H}} 0,97(J=6,9 \mathrm{~Hz})$.

Conforme verificado no espectro de ${ }^{1} \mathrm{H}-{ }^{1} \mathrm{H}$ COSY, o hidrogênio heterotópico $\mathrm{H}-2 \mathrm{a}$ correlaciona com o H-3 e H-2b correlaciona com H-1, sendo que estes também correlacionam entre si. Outra correlação importante é observada para H-11 que se correlaciona com os hidrogênios H-12, H-13 e H-4.

Só foi possível definir a posição da hidroxila $\left(\delta_{\mathrm{H}}\right.$ $4,89)$ com o espectro de diferença de NOE que através da irradiação no sinal $\delta_{\mathrm{H}} 1,19(\mathrm{H}-14)$ apresentou aumento na intensidade dos sinais dos hidrogênios $\delta_{\mathrm{H}} 3,07(\mathrm{H}-1), \delta_{\mathrm{H}}$ $4,89(\mathrm{OH}), \delta_{\mathrm{H}} 1,50(\mathrm{H}-2 \mathrm{a})$ e $\delta_{\mathrm{H}} 1,78(\mathrm{H}-3 \mathrm{a})$ e através da irradiação no sinal $\delta_{\mathrm{H}} 3,07(\mathrm{H}-1)$ apresentou aumento na intensidade dos sinais dos hidrogênios $\delta_{\mathrm{H}} 1,19$ (H-14), $\delta_{\mathrm{H}} 4,89(\mathrm{OH}), \delta_{\mathrm{H}} 1,50(\mathrm{H}-2 \mathrm{a})$ e $\delta_{\mathrm{H}} 1,96(\mathrm{H}-2 \mathrm{~b})$. Diante dessas informações e comparação com dados da literatura (Mulholland et al., 1998) foi possível determinar que a hidroxila está ligada ao C-8, e que as metilas 13, 14 e 15, na publicação de Mulholland et al. (1998), estão com $\delta_{C}$ trocados, devendo serem corrigidos para $\delta_{\mathrm{C}} 22,3(13), \delta_{\mathrm{C}}$ $21,4(14)$ e $\delta_{C} 21,3(15)$. Desta forma, a substância isolada 1 foi identificada como sendo o sesquiterpeno: 8-hidróxicalameneno.

A mistura das substâncias $\mathbf{2 a}$ e $\mathbf{2 b}$ (cristais brancos, pouco solúveis em hexano e totalmente solúveis em $\mathrm{CH}_{2} \mathrm{Cl}_{2}$.) foi isolada da fração hexânica das folhas de $C$. psidiiflorus. A concordância entre os dados de RMN de ${ }^{1} \mathrm{H}$, RMN de ${ }^{13} \mathrm{C}$, para as estruturas propostas, e comparação com os dados de $\mathrm{RMN}$ de ${ }^{13} \mathrm{C}$ da literatura (Mahato \& Kundu, 1994), possibilitou a identificação das substâncias isoladas $\mathbf{2 a}$ e $\mathbf{2 b}$ como mistura dos triterpenos $\alpha$-amirina (2a) e $\beta$-amirina (2b).

A substância 3 (sólido amarelo fluorescente em
UV $254 \mathrm{~nm}$ ) foi isolada da fração acetato de etila, das folhas de $C$. psidiiflorus. Através do espectro de RMN de ${ }^{1} \mathrm{H}$ foi possível identificar a unidade aglicônica da substância 3 como canferol, devido à presença de dois dubletos em $\delta_{\mathrm{H}}$ 6,32 e $\delta_{H} 6,15$, com integração para um hidrogênio cada, e com padrão de acoplamento meta $(J=1,8 \mathrm{~Hz})$ e dois dubletos em $\delta_{\mathrm{H}} 6,92$ e $\delta_{\mathrm{H}} 7,75$, com integração para dois hidrogênios cada, e com acoplamento orto $(J=9,0 \mathrm{~Hz})$. Os espectros de RMN de ${ }^{13} \mathrm{C}$ e o DEPT confirmaram a unidade aglicônica como canferol, pela presença de uma carbonila em $\delta_{\mathrm{C}} 179,58$ e sinais de carbonos na região de aromáticos, correspondentes aos quinze carbonos da unidade aglicona.

A presença de duas unidades glicosídicas foi evidenciada pelos sinais de dois hidrogênios anoméricos em $\delta_{\mathrm{H}} 4,25(\mathrm{~d}, J=7,5 \mathrm{~Hz})$ e $\delta_{\mathrm{H}} 5,35(\mathrm{~d}, J=1,8 \mathrm{~Hz})$ correlacionados aos carbonos anoméricos em $\delta_{\mathrm{C}} 104,34 \mathrm{e}$ $\delta_{\mathrm{C}} 103,52$, respectivamente, de acordo com o observado no espectro de HMQC. Uma das unidades glicosídicas foi caracterizada como glicose devido à presença de um carbono oximetilênico em $\delta_{\mathrm{C}} 62,75$, um hidrogênio anomérico com constante de acoplamento $(J=7,5 \mathrm{~Hz})$ caracterizando configuração $\beta$ para o carbono anomérico e demais sinais de carbonos oximetínicos. A outra unidade glicosídica foi caracterizada como raminose, devido à presença de um grupo metílico em $\delta_{\mathrm{H}} 0,91$ (d, $J=6,0 \mathrm{~Hz}$ ) correlacionando ao sinal $\delta_{\mathrm{C}} 17,64$, de acordo com o observado no espectro de HMQC, e a presença do hidrogênio anomérico com constante de acoplamento ( $J$ $=1,8 \mathrm{~Hz}$ ) caracterizando configuração $\alpha$ para o carbono anomérico, e acoplamento do tipo axial-equatorial entre o H-anomérico H-1", com H-2",.

A posição de ligação da raminose foi determinada pela análise do espectro de HMBC devido a correlação entre o sinal do hidrogênio anomérico $\delta_{\mathrm{H}} 5,35$ (H-1"') e o $\delta_{\mathrm{C}}$ 136,17 (C-3) da unidade aglicônica. A concordância entre os dados de RMN de ${ }^{1} \mathrm{H}, \mathrm{RMN}$ de ${ }^{13} \mathrm{C}$, DEPT $135^{\circ}$ e $90^{\circ}$, HMQC $\left({ }^{1} \mathrm{H} \mathrm{x}{ }^{13} \mathrm{C}\right)$ e HMBC ${ }^{3} \mathrm{~J}\left({ }^{1} \mathrm{H} \mathrm{x}{ }^{13} \mathrm{C}\right)$ para a estrutura proposta e comparação com os dados de RMN de ${ }^{1} \mathrm{H}$ da literatura (Sharafet al., 1997), possibilitou a identificação da substância isolada 3 como 3-O- $\alpha$-ramnopiranosil-7-O$\beta$-glicopiranosil canferol.

A substância 4 foi isolada por extração ácidobase, dos galhos de C. psidiiflorus. No espectro de RMN de ${ }^{1} \mathrm{H}$ foram observados sinais na região de hidrogênios aromáticos, em $\delta_{\mathrm{H}} 6,96\left(\mathrm{td}, J=7,8\right.$ e 1,2 Hz), $\delta_{\mathrm{H}} 6,98(\mathrm{td}$, $J=7,2$ e $1,2 \mathrm{~Hz}), \delta_{\mathrm{H}} 7,27(\mathrm{dd}, J=7,8$ e $0,9 \mathrm{~Hz})$ e $\delta_{\mathrm{H}} 7,37$ (dd, $J=7,5$ e $0,9 \mathrm{~Hz}$ ), cada um apresentando integração para um hidrogênio. Além destes, foram observados um quarteto em $\delta_{\mathrm{H}} 4,15(J=6,6 \mathrm{~Hz})$, com integração para um hidrogênio; um multipleto em $\delta_{\mathrm{H}} 2,99$, com integração para dois hidrogênios e um dubleto em $\delta_{\mathrm{H}} 1,48(J=6,6$ $\mathrm{Hz})$, correspondente a um grupo metila. Em $\delta_{\mathrm{H}} 1,48(J=$ $6,6 \mathrm{~Hz}$ ) foi verificado um dubleto, com integração para três hidrogênios, característico de metila.

No espectro de $\mathrm{RMN}$ de ${ }^{13} \mathrm{C}$ foram observados 
onze picos e através de DEPT $135^{\circ}$ e $90^{\circ}$ foi possível verificar que sete destes carbonos estavam ligados a hidrogênio: uma metila $\left(\delta_{\mathrm{C}} 18,99\right)$, dois metilenos $\left(\delta_{\mathrm{C}}\right.$ 42,36 e $\left.\delta_{C} 21,59\right)$ e quatro carbonos aromáticos $\left(\delta_{C} 110,61\right.$, $\delta_{\mathrm{C}} 120,76, \delta_{\mathrm{C}} 118,44$ e $\left.\delta_{\mathrm{C}} 117,37\right)$. Através do HMQC $\left({ }^{1} \mathrm{H}\right.$ $\mathrm{x}{ }^{13} \mathrm{C}$ ) foi possível atribuir o valor do décimo segundo carbono $\left(\delta_{\mathrm{C}} 48,50\right)$ que no espectro de $\mathrm{RMN}{ }^{13} \mathrm{C}$ ficou sob o sinal do solvente.

Através do COSY $\left({ }^{1} \mathrm{H} \mathrm{x}{ }^{1} \mathrm{H}\right)$ foi possível verificar as correlações entre $\delta_{\mathrm{H}} 7,27(\mathrm{H}-5)$ e $\delta_{\mathrm{H}} 6,98(\mathrm{H}-6), \delta_{\mathrm{H}} 6,96$ $(\mathrm{H}-7)$ e $\delta_{\mathrm{H}} 7,37(\mathrm{H}-8)$ e entre os hidrogênios $\delta_{\mathrm{H}} 6,98(\mathrm{H}-6)$ e $\delta_{\mathrm{H}} 6,96(\mathrm{H}-7)$.

A concordância entre os dados de $\mathrm{RMN}$ de ${ }^{1} \mathrm{H}$, RMN de ${ }^{13} \mathrm{C}$, DEPT $135^{\circ}$ e $90^{\circ}$, COSY $\left({ }^{1} \mathrm{H} \mathrm{x}{ }^{1} \mathrm{H}\right)$, HMQC $\left({ }^{1} \mathrm{H} \mathrm{x}{ }^{13} \mathrm{C}\right)$ para a estrutura proposta e comparação com os dados da literatura (Ortet, 2003), possibilitou a identificação da substância isolada 4 como sendo o alcaloide 1,2,3,4tetraidro-1-metil- $\beta$-carbolina (eleagenina).

A avaliação da toxicidade frente ao microcrustáceo Artemia salina mostrou que o extrato bruto das folhas de $C$. psidiiflorus foi mais ativo que o extrato bruto dos galhos, $\mathrm{DL}_{50}(\mu \mathrm{g} / \mathrm{mL})=186,64$ e 566,24, respectivamente Dentre as frações de folhas e galhos, a que apresentou maior toxicidade foi a fração clorofórmica dos galhos de
C. psidiiflorus $\left(\mathrm{DL}_{50}=84,91 \mu \mathrm{g} / \mathrm{mL}\right.$ e $\mathrm{DL}_{90}=746,44 \mu \mathrm{g} /$ $\mathrm{mL})$.

$\mathrm{Na}$ avaliação da atividade antibacteriana os extratos brutos e as frações acetato de etila e $n$-butanol das folhas e galhos de C. psidiiflorus apresentaram-se "moderadamente ativas" frente às bactérias Staphylococcus aureus e Bacillus subtilis. A substância isolada 1 (8-hidróxicalameneno) apresentou-se "fortemente ativa" frente às bactérias $S$. aureus $(\mathrm{CMI}=7,8 \mu \mathrm{g} / \mathrm{mL}$ e $\mathrm{CMB}=15,6 \mu \mathrm{g}$ / $\mathrm{mL}$ ) e $B$. subtilis (CMI e CMB $=7,8 \mu \mathrm{g} / \mathrm{mL}$ ).

$\mathrm{Na}$ avaliação da atividade antifúngica, dentre os extratos brutos das folhas e dos galhos, e suas frações, o melhor resultado foi observado para a fração hexânica das folhas de C. psidiiflorus que se apresentou "ativa" (CMI $=62,5 \mu \mathrm{g} / \mathrm{mL}$ ) frente ao fungo Candida parapsilosis e "moderadamente ativa" frente aos fungos Candida albicans e Candida tropicalis. As folhas apresentaram melhores resultados de atividade antifúngica que os galhos de Calycorectes psidiiflorus. A substância isolada 1 (8-hidróxi-calameneno) apresentou-se "mais ativa" frente aos fungos Candida albicans, Candida parapsilosis e Candida tropicalis $(\mathrm{CMI}=15,6 \mu \mathrm{g} / \mathrm{mL})$ e a substância isolada $4 \quad(1,2,3,4$-tetraidro-1-metil- $\beta$-carbolina) apresentou-se "moderadamente ativa" frente ao fungo Candida tropicalis.<smiles>Cc1cc(O)c2c(c1)[C@@H](C(C)C)CC[C@H]2C</smiles>

1



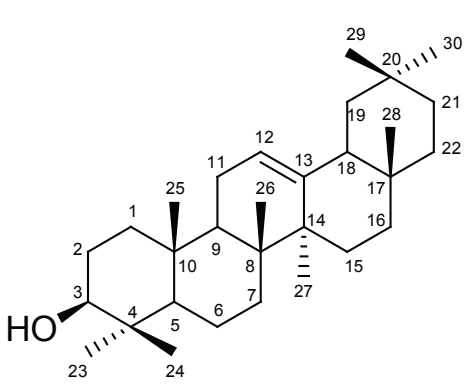

2b

\section{AGRADECIMENTOS}

Domingues).

À CAPES pela bolsa de mestrado (Elaine A.

\section{REFERÊNCIAS}

Dey PM, Harbone JB 1993. Methods in plant biochemistry (assays for bioactivity). Ed. Academic Press 6: 7-10.
Mahato SB, Kundu AP 1994. ${ }^{13} \mathrm{C}$ NMR Spectra of pentacyclic triterpenoids-A compilation and some salient features. Phytochemistry 37: 1517-1575.

Mclaughlin JL, Chang CJ, Smith DL 1991. Studies in natural products chemistry. Ed. Atta Rahman 9: 383-409.

Mulholland DA, Iourine S, Taylor DAH 1998. Sesquiterpenoids from Dysoxylum schiffneri. Phytochemistry 47: 14211422.

Ortet MRLBV 2003. Estudo Químico da espécie vegetal Eugenia 
repanda Berg. (Myrtaceae). Maringá. Dissertação de mestrado, Programa de Pós-Graduação em Química, Universidade Estadual de Maringá.

Romagnolo MB 2003. A família Myrtaceae na planicie alagável do alto rio Paraná, estados de Mato Grosso do Sul e Paraná, Brasil. Maringá, 120p. Tese de doutorado, Universidade Estadual de Maringá.

Schmeda HG 1995. Flavonoids from Calycorectes, Campomanesia, Eugenia e Hexachlamys species. Fitoterapia 66: 373-374.

Sharaf M, El-Ansari MA, Saleh NAM 1997. Flavonoids of four cleome and three capparis species. Biochem Syst Ecol 25: 161-166.

Trease GE 1983. Pharmacognosy. 12 ed. London:. 